\title{
Analysis of shot noise suppression for electron beams
}

\author{
Daniel Ratner \\ Department of Applied Physics, Stanford University, Stanford, California 94305, USA
}

Zhirong Huang and Gennady Stupakov

SLAC, Menlo Park, California 94309, USA

(Received 14 August 2010; published 24 June 2011)

\begin{abstract}
Shot noise can affect the performance of free-electron lasers (FELs) by driving instabilities (e.g., the microbunching instability) or by competing with seeded density modulations. Recent papers have proposed suppressing shot noise to enhance FEL performance. In this paper we use a onedimensional (1D) model to calculate the noise amplification from an energy modulation (e.g., electron interactions from space charge or undulator radiation) followed by a dispersive section. We show that, for a broad class of interactions, selecting the correct dispersive strength suppresses shot noise across a wide range of frequencies. The final noise level depends on the beam's energy spread and the properties of the interaction potential. We confirm and illustrate our analytical results with 1D simulations.
\end{abstract}

DOI: $10.1103 /$ PhysRevSTAB.14.060710

PACS numbers: 29.27.- a, 41.60.Dk, 41.60.Cr, 07.50.Hp

\section{INTRODUCTION}

In a bunch of random (uncorrelated) electrons, the longitudinal density contains white noise fluctuations, commonly called shot noise. While shot noise drives self-amplified spontaneous emission (SASE) free-electron lasers (FELs), the same density fluctuations may adversely affect FEL operation. For example, the microbunching instability, thought to originate from shot noise, can incapacitate diagnostics and degrade FEL performance [1-7]. Shot noise also competes with external modulations in the operation of seeded FELs [8,9]. Recent papers have proposed schemes to decrease the noise level below that of shot noise to aid the FEL process or for other applications [10-12]. In this paper we use the approach of [13] to study the evolution of noise as the beam travels through a system with interactions between the electrons as well as dispersive regions. To simplify the analysis, we consider a one-dimensional (1D) model system of a generic selfinteraction $h$, which changes the particle energies, followed by a dispersive region $R_{56}$, which converts the change in energy to change in position (Fig. 1). We show that, for a broad class of interactions, it is possible to suppress density fluctuations below the shot noise level, and we provide 1D simulations to confirm the result. Future theoretical and numerical work will extend these results to 3D models, and explore the feasibility of demonstrating shot noise suppression experimentally.

Published by the American Physical Society under the terms of the Creative Commons Attribution 3.0 License. Further distribution of this work must maintain attribution to the author(s) and the published article's title, journal citation, and DOI.

\section{ANALYTICAL MODEL}

\section{A. Noise factor}

To characterize the level of noise at a wave vector $k$, we define the noise factor

$$
F(k, s) \equiv \frac{1}{N} \sum_{j, l} e^{i k\left[z_{j}(s)-z_{l}(s)\right]},
$$

where $z_{j}(s)$ is the longitudinal bunch coordinate of particle $j$ at position $s$ in the accelerator, and $N$ is the number of particles in the beam. We note that the noise factor can equivalently be defined by $F(k, s) \equiv N|b(k, s)|^{2}$, with the bunching factor $b(k, s) \equiv \sum_{j} \exp \left[i k z_{j}(s)\right] / N$.

The noise factor $F(k, s)$ is a measure of the correlations between particle coordinates at wave vector $k$. If the particle positions are uncorrelated, we find the expectation value of shot noise, $\langle F(k, s)\rangle=1$. On the other hand, if the positions are strongly correlated at wave vector $k$, we find $\langle F(k, s)\rangle \sim N$, with $N \gg 1$ generally; such correlated (or "bunched") beams are found at the output of an FEL, and as the result of the microbunching instability [6,7]. We may also consider the case of an anticorrelated (or "quiet") beam, with $\langle F(k, s)\rangle<1$, below the shot noise level. In this

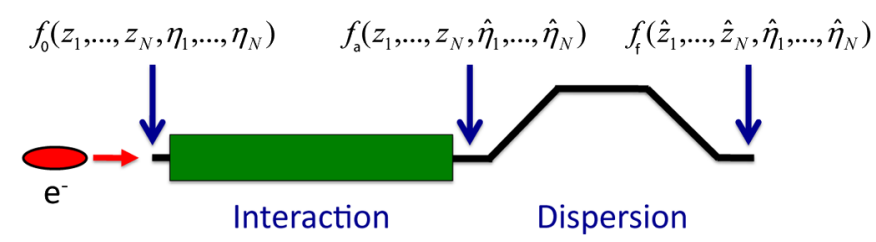

FIG. 1. Schematic of our model system. Starting with an initial electron distribution function $f_{0}(z, \eta)$, the interaction and dispersive regions produce a final distribution function $f_{f}(\hat{z}, \hat{\eta})$. The dispersion may be positive or negative. 
paper, we investigate the possibility of producing quiet beams.

Though the noise factor is defined as a function of accelerator position $s$, we are particularly interested in the noise level at the output of our system, $F\left(k, s_{f}\right)$. Starting from an initial distribution function at $s_{0}$, we would like to determine the resulting final noise level at $s_{f}$.

To facilitate an analytical solution, we will study the simplified system of Fig. 1. We assume the particle distribution is a function of position in the bunch, $z(s)$, and relative, normalized energy, $\eta(s) \equiv\left[E(s)-E_{0}\right] / E_{0}$, with average beam energy $E_{0}$. Though both $z$ and $\eta$ are functions of $s$, we are primarily interested in the initial and final coordinates, so for brevity we define $z, \eta \equiv$ $z\left(s_{0}\right), \eta\left(s_{0}\right), \hat{z}, \hat{\eta} \equiv z\left(s_{f}\right), \eta\left(s_{f}\right)$, and $F(k) \equiv F\left(k, s_{f}\right)$. We can then describe the system as follows. We start with a simple $N$-particle initial distribution of particles, $f_{0}\left(z_{1}, \ldots, z_{N}, \eta_{1}, \ldots, \eta_{N}\right)$. After an interaction period, the energies are modified, giving distribution $f_{a}\left(z_{1}, \ldots, z_{N}\right.$, $\hat{\eta}_{1}, \ldots, \hat{\eta}_{N}$ ). A dispersive region (assumed to have zero interaction), then changes the longitudinal positions, giving final distribution $f_{f}\left(\hat{z}_{1}, \ldots, \hat{z}_{N}, \hat{\eta}_{1}, \ldots, \hat{\eta}_{N}\right)$.

\section{B. Expectation value of noise factor}

To calculate the expectation value, we break $\langle F(k)\rangle$ into incoherent $(j=l)$ and coherent $(j \neq l)$ portions. First, we treat the incoherent portion. With $j=l$, the phases cancel and we find $N$ terms, all equal to 1 , giving

$$
\langle F(k)\rangle_{S N}=1,
$$

which is simply the noise level due to shot noise.

Next, we calculate the coherent portion. To find the expectation value at the final accelerator position, we integrate $F(k)$ over the final particle distributions, $f_{f}\left(\hat{z}_{1}, \ldots, \hat{z}_{N}, \hat{\eta}_{1}, \ldots, \hat{\eta}_{N}\right)$. In general, $f_{f}$ may be a complicated function of all $2 N$ variables. However, if we assume the electrons are initially uncorrelated, then we can write the initial distribution function as

$$
f_{0}\left(z_{1}, \ldots, z_{N}, \eta_{1}, \ldots, \eta_{N}\right)=\prod_{i}^{N} f^{(1)}\left(z_{i}, \eta_{i}\right)
$$

with the single particle distribution functions for a beam with Gaussian energy spread of $\sigma_{\eta}$ and uniform longitudinal density of length $L$ given by

$$
f^{(1)}(z, \eta)= \begin{cases}\frac{e^{-\eta^{2} / 2 \sigma_{\eta}^{2}}}{\sqrt{2 \pi} \sigma_{\eta} L} & \text { for }-L / 2<z<L / 2 \\ 0 & \text { elsewhere. }\end{cases}
$$

We then express the final coordinates in terms of the initial coordinates $(\hat{z}, \hat{\eta} \rightarrow z, \eta)$, and integrate over the product of $N$ simple initial distributions, $f^{(1)}$.

In the interaction region, we assume the bunch is longitudinally frozen $(z, \eta \rightarrow z, \hat{\eta})$, and likewise in the dispersive region we assume there is zero interaction $(z, \hat{\eta} \rightarrow \hat{z}, \hat{\eta})$. To further simplify the calculation, we ignore any transverse effects. (The validity of the 1D approximation will depend on the interaction of interest.) Our resulting map from initial to final coordinates then is

$$
\eta_{j} \rightarrow \hat{\eta}_{j}=\eta_{j}+\sum_{i=1}^{N} h\left(z_{j}, z_{i}\right) \quad z_{j} \rightarrow \hat{z}_{j}=z_{j}+R_{56} \hat{\eta}_{j}
$$

with dispersive strength $R_{56}$, and $h\left(z_{j}, z_{i}\right)$ the change in energy of particle $j$ due to the interaction with particle $i$. We can now write the coherent portion of $F(k)$ in terms of the initial coordinates, and integrate over each single particle distribution, $f_{i} \equiv f^{(1)}\left(z_{i}, \eta_{i}\right)$, to find the expectation value,

$$
\begin{aligned}
& \langle F(k)\rangle_{C} \approx N \int_{-L / 2}^{L / 2} d z_{1} d \eta_{1} \cdots \int_{-L / 2}^{L / 2} d z_{N} d \eta_{N} f_{1} \cdots \\
& \times f_{N} e^{i k\left\{z_{1}-z_{2}+R_{56}\left(\eta_{1}-\eta_{2}\right)+R_{56}\left[\sum_{i}^{N} h\left(z_{1}, z_{i}\right)-\sum_{m}^{N} h\left(z_{2}, z_{m}\right)\right]\right\}},
\end{aligned}
$$

where we have assumed the $N^{2}-N \approx N^{2} \gg 1$ coherent terms of the sum in Eq. (1) are identical, and we have chosen $j=1, l=2$ without loss of generality.

Our approach (following [13]) will be to explicitly separate the $z_{1}, z_{2}$ terms. We assume the interaction depends only on the distance between the particles, $h\left(z_{1}, z_{2}\right)=$ $h\left(z_{1}-z_{2}\right)$, so we change variables, $z_{1}, z_{2}, z_{l}, z_{m} \rightarrow$ $\zeta, Z, \tau_{l}, \tau_{m}$ with $\zeta \equiv z_{1}-z_{2}, Z \equiv\left(z_{1}+z_{2}\right) / 2$, and $\tau_{l, m} \equiv$ $z_{l, m}-z_{2}$. Finally, we assume that the interaction is nonzero only within a characteristic distance $L_{h}$, which is much shorter than the bunch length $L$. We can then integrate over $Z$ and $\eta_{1} \ldots \eta_{N}$ to find

$$
\langle F(k)\rangle_{C}=n_{0} e^{-k^{2} R_{56}^{2} \sigma_{\eta}^{2}} \int_{-\infty}^{\infty} d \zeta\left[\frac{1}{L^{N-2}} \int d \tau_{3} \cdots \int d \tau_{N} e^{i k\left\{\zeta+R_{56}[h(\zeta)-h(-\zeta)]+R_{56} \sum_{i=3}^{N}\left[h\left(-\tau_{i}+\zeta\right)-h\left(-\tau_{i}\right)\right]\right\}}\right],
$$

where we have defined the $1 \mathrm{D}$ particle density $n_{0} \equiv N / L$ and we have used $L_{h} \ll L$ to both ignore edge effects and set the $\zeta$ integral limits to infinity. First, we note that the $N-2$ integrals over $\tau_{i}$ are separable and identical. Second, we assume $k R_{56} h \ll 1$ so we can linearize the exponentials, yielding

$$
\langle F(k)\rangle_{C} \approx n_{0} e^{-k^{2} R_{56}^{2} \sigma_{\eta}^{2}} \int_{-\infty}^{\infty} d \zeta e^{i k \zeta}\left[1+\Gamma_{1}(\zeta)\right]\left[1+\frac{1}{N} \Gamma_{2}(\zeta)\right]^{N-2},
$$

with definitions 


$$
\begin{aligned}
\Gamma_{1}(\zeta) \equiv & i k R_{56}[h(\zeta)-h(-\zeta)]+\cdots \\
\Gamma_{2}(\zeta) \equiv & n_{0} \int_{-\infty}^{\infty} d \tau\left(i k R_{56}[h(-\tau+\zeta)-h(-\tau)]\right. \\
& \left.-\frac{k^{2} R_{56}^{2}}{2}[h(-\tau+\zeta)-h(-\tau)]^{2}+\cdots\right),
\end{aligned}
$$

where we have expanded $\Gamma_{1}$ and $\Gamma_{2}$ in powers of the small parameter $k R_{56} h$. For $\Gamma_{1}$, the term linear in $k R_{56} h$ is nonzero, so we drop all higher order terms. However, from our assumption of a long bunch, the linear order terms in $\Gamma_{2}$ cancel after the integration, so we must also keep the quadratic term for $\Gamma_{2}$. Combining the two square terms, $\int d \tau h(-\tau)^{2}=\int d \tau h(-\tau+\zeta)^{2}$, we find

$$
\begin{aligned}
& \Gamma_{1}(\zeta) \approx i k R_{56}[h(\zeta)-h(-\zeta)] \\
& \Gamma_{2}(\zeta) \approx n_{0} k^{2} R_{56}^{2} \int_{-\infty}^{\infty} d \tau\left[h(-\tau+\zeta) h(-\tau)-h(-\tau)^{2}\right] .
\end{aligned}
$$

We may be tempted to drop $\Gamma_{2}$, because it is second order in $k R_{56} h$. However, $\Gamma_{2}$ is also raised to the power of $N$, and with $N \gg 1$ generally, $\Gamma_{2}$ may even be the dominant term (as for the microbunching instability, see e.g. [6,7]). In this paper, we keep both terms, and will see that noise suppression occurs when $\Gamma_{1}$ and $\Gamma_{2}$ are comparable.

\section{Analytical expression: Weak interaction}

If we consider a weak interaction under the stronger assumption $\Gamma_{2} \ll 1$, we can solve for the noise level analytically. Adding in the shot noise term again and expanding Eq. (8), we find

$$
\begin{aligned}
\langle F(k)\rangle \approx & 1+n_{0} e^{-k^{2} R_{56}^{2} \sigma_{\eta}^{2}}\left\{\int_{-\infty}^{\infty} d \zeta e^{i k \zeta\left[\Gamma_{1}(\zeta)+\Gamma_{2}(\zeta)\right]}\right. \\
& +2 \pi \delta(k)\} .
\end{aligned}
$$

We are interested in $k \neq 0$, and so will drop the $\delta$ function. (The $\delta$ function arises from our assumption of $L \rightarrow \infty$. For finite $L$, we will have a term that is nonzero for $k<1 / L$, but even so our focus is on much shorter wavelengths.)

We can now identify the three regimes for $\langle F(k)\rangle$. For zero interaction, we are left with only the leading shot noise term $\langle F(k)\rangle=1$, which is simply the white noise of an uncorrelated bunch. The $\Gamma_{2}$ contribution is positive definite, so for $\Gamma_{2} \gg \Gamma_{1}$, we find a correlated beam with $\langle F(k)\rangle>1$. Finally, for $\Gamma_{1} \sim \Gamma_{2}$, the term linear in $R_{56}$ cannot be neglected. If $R_{56}$ is chosen so that $\Gamma_{1}<0$, it is possible to create an anticorrelated beam, with the noise factor suppressed below the shot noise level $\langle F(k)\rangle<1$. In this paper we consider the third regime.

Identifying the $\zeta$ integral as a Fourier transform (FT), we rewrite the noise factor as

$$
\begin{aligned}
\langle F(k)\rangle \approx & 1+i n_{0} k R_{56}[\tilde{h}(k)-\tilde{h}(-k)] e^{-k^{2} R_{56}^{2} \sigma_{\eta}^{2}} \\
& +n_{0}^{2} k^{2} R_{56}^{2} \mathrm{FT}\left\{\int_{-\infty}^{\infty} d \tau[h(-\tau) h(-\tau+\zeta)\right. \\
& \left.\left.-h(-\tau)^{2}\right]\right\} e^{-k^{2} R_{56}^{2} \sigma_{\eta}^{2}},
\end{aligned}
$$

where $\tilde{h}(k)$ denotes $\operatorname{FT}\{h(\tau)\}$. We drop the second term in the remaining integral because it has no $\zeta$ dependence, and so its Fourier transform is nonzero only for wavelengths longer than the bunch $(k<1 / L)$. The first term is the autocorrelation of $h(\tau)$, which has Fourier transform $|\tilde{h}(k)|^{2}$, yielding

$$
\begin{aligned}
\langle F(k)\rangle \approx & 1-2 n_{0} k R_{56} \operatorname{Im}[\tilde{h}(k)] e^{-k^{2} R_{56}^{2} \sigma_{\eta}^{2}} \\
& +n_{0}^{2} k^{2} R_{56}^{2}|\tilde{h}(k)|^{2} e^{-k^{2} R_{56}^{2} \sigma_{\eta}^{2}} .
\end{aligned}
$$

If the energy spread is small $\left(\sigma_{\eta} \rightarrow 0\right)$, and the interaction has purely imaginary Fourier transform $\tilde{h}(k)$, we can write the noise factor as a perfect square

$$
\langle F(k)\rangle \approx(1-\Upsilon)^{2} \quad \text { with } \Upsilon \equiv n_{0} k R_{56} \operatorname{Im}[\tilde{h}(k)] .
$$

We suppress the noise factor below the shot noise level when the suppression parameter is in the range $0<Y<2$ and the noise disappears completely for $Y=1$. (We note that partial noise suppression is possible even if the interaction contains a real component.) We are particularly interested in interactions that can be approximated as step functions near $\zeta=0: h(\zeta) \rightarrow A H(\zeta)+$ const, with Heaviside function $H$, and interaction strength $A$. For such interactions, we find $\tilde{h}(k) \propto 1 / k$ for high frequencies, so that $Y$ is independent of $k$. We are then able to simultaneously suppress bunching at a wide range of frequencies.

We can draw a broad lesson from Eq. (13); a quiet beam is attainable from any interaction with primarily imaginary Fourier transform, e.g., from step-function interactions (for $k \neq 0$ ). We will treat the special cases of space charge and undulator interactions later, but here emphasize that any interaction with imaginary Fourier transform will suffice. For example, the wake from a linac with periodic structures also satisfies these conditions [14]. We have assumed negligible energy spread here; see Appendix $\mathrm{C}$ for a discussion of the effect of energy spread on noise suppression.

For a physical interpretation of the requirement for imaginary Fourier transform, we consider a test particle in front of localized density spike of width $1 / k$. If $h(\tau)>0$ for $\tau>0$, the test particle will receive positive energy change. A positive dispersive region then causes the test particle to move forward and away from the dense region. Likewise, a test particle at the back of a dense region $(\tau<0)$ loses energy relative to the front particle for $\tilde{h}(k)$ imaginary, and moves backward and away in a positive dispersive region. The end result is a reduction in the density spike and thus a reduction in the noise. If $h(\tau)<0$ for $\tau>0$, as is the case for an undulator, we have the identical argument, but 


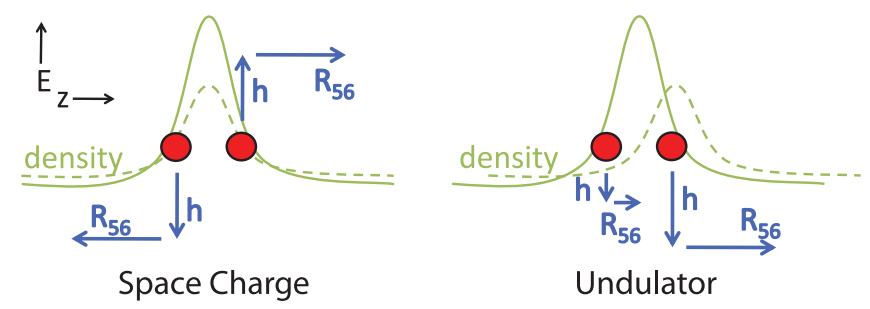

FIG. 2. Schematic of an interaction near a density spike (solid green line). At left, for the space charge case, particles in the front half of the spike gain energy, while particles in the back half lose energy, and in positive dispersion, the density spike shrinks (dotted green line). We have a similar result for an interaction due to undulator radiation (right). At high frequency (spike much shorter than undulator resonant wavelength), all particles lose energy, but following a dispersive region with negative $R_{56}$ we still find a reduction in the density spike (dotted green line).

require negative dispersion. The process is illustrated in Fig. 2.

\section{Numerical approximation: Strong interaction}

For stronger interactions, we may not be able to approximate $\Gamma_{2} \ll 1$. If it is not possible to evaluate Eq. (8) analytically for an arbitrary $h$, we can carry out the integrals numerically. Using the less stringent approximation $\Gamma_{2} \ll N$ (satisfied even for simulation parameters with relatively small $N)$, we take $\left(1+\Gamma_{2} / N\right)^{N} \approx \exp \left(\Gamma_{2}\right)$ to obtain

$$
\langle F(k)\rangle=1+n_{0} e^{-k^{2} R_{56}^{2} \sigma_{\eta}^{2}} \int_{-\infty}^{\infty} d \zeta e^{i k \zeta} e^{\Gamma_{2}(\zeta)}\left[1+\Gamma_{1}(\zeta)\right] .
$$

For physical interactions, $\Gamma_{1} \rightarrow 0$ as $\zeta \rightarrow \infty$, so the second term, $e^{\Gamma_{2}(\zeta)} \Gamma_{1}(\zeta)$, converges and can be integrated numerically. We cannot directly integrate the first term $\exp \left[\Gamma_{2}(\zeta)\right]$, because the $h^{2}(-\tau)$ in Eq. (10) has no $\zeta$ dependence; in the limit $\zeta \rightarrow \infty$, we find $\Gamma_{2}(\zeta) \rightarrow \bar{\Gamma}_{2} \neq 0$, and the integral diverges. However, the divergence occurs only for $k=0$; otherwise, $\bar{\Gamma}_{2} \exp [i k \zeta]$ integrates to zero [which is why we dropped the $h^{2}(-\tau)$ term from Eq. (12)]. Following the same reasoning here, with

$$
\bar{\Gamma}_{2}=-n_{0} k^{2} R_{56}^{2} \int_{-\infty}^{\infty} d \tau h(-\tau)^{2},
$$

we explicitly remove the constant term $\exp \left[\bar{\Gamma}_{2}\right]$ to find

$$
\begin{aligned}
\langle F(k)\rangle= & 1+n_{0} e^{-k^{2} R_{56}^{2} \sigma_{\eta}^{2}} \int_{-\infty}^{\infty} d \zeta\left[\left(e^{\Gamma_{2}(\zeta)}-e^{\bar{\Gamma}_{2}}\right) \cos (k \zeta)\right. \\
& \left.+i e^{\Gamma_{2}(\zeta)} \Gamma_{1}(\zeta) \sin (k \zeta)\right]
\end{aligned}
$$

where we have used Eq. (10) to see that $\Gamma_{1}(\zeta)$ and $\Gamma_{2}(\zeta)$ are, respectively, odd and even functions of $\zeta$. We can then integrate Eq. (17) numerically.

\section{SPACE CHARGE CASE}

\section{A. Space charge interaction}

So far we have not specified the interaction term, constraining only that the energy change $h$ is a function of $\zeta$, the distance between the particles. We now consider the Coulomb interaction between two particles. We assume the interaction occurs over a distance $L_{a}$ in the accelerator, during which the particles are frozen longitudinally. We consider a 1D system, treating the particles as uniform, rigid sheets of charge with radius $a$, valid in the limit $a \gg$ $\gamma / k$ [15]. To calculate the relative change in energy due to the longitudinal $E$ field $\left(E_{z}\right)$, we integrate over the sheets of source and test particles,

$$
\begin{aligned}
h_{s c}(\zeta)= & \frac{\operatorname{sgn}(\zeta)}{\gamma m_{e} c^{2}} \frac{q^{2} L_{a}}{4 \pi \epsilon_{0} S^{2}} \int_{0}^{a} \int_{0}^{a} \int_{0}^{2 \pi} \int_{0}^{2 \pi} \\
& \times \frac{r_{1} d r_{1} r_{2} d r_{2} d \theta_{1} d \theta_{2}(\gamma \zeta)}{\left[(\gamma \zeta)^{2}+r_{1}^{2}+r_{2}^{2}-2 r_{1} r_{2} \cos \left(\theta_{1}-\theta_{2}\right)\right]^{3 / 2}},
\end{aligned}
$$

with average particle energy $\gamma m c^{2}$, electron charge $e$, area of sheet $S=\pi a^{2}$, and

$$
\operatorname{sgn}(\zeta) \equiv \begin{cases}1 & \zeta>0 \\ 0 & \zeta=0 \\ -1 & \zeta<0\end{cases}
$$

One of the $\theta$ integrals trivially gives a factor of $2 \pi$, and the remaining integrals can be solved numerically to produce the interaction $h_{s c}(\zeta)$ shown in Fig. 3. We note that the interaction will go to zero for $\zeta \gg a / \gamma$, as required in our derivation of Eq. (7). In the limit of infinite sheets $(a \rightarrow \infty)$, the $E_{z}$ field is simply

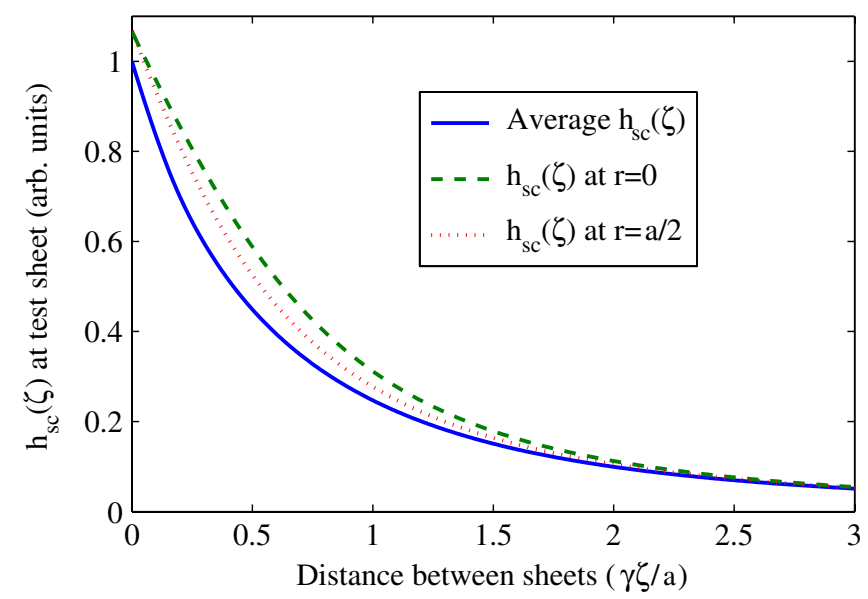

FIG. 3. Space charge from a source sheet produces a change in energy $\left(h_{s c}\right)$ in a test sheet located at a distance $\zeta$. We calculate the energy modulation to the test sheet by averaging over the entire sheet (solid blue curve). Though $E_{z}$ (and thus $h_{s c}$ ) is not constant everywhere in the test sheet, we note that there is relatively little variation near the center of the sheet, as can be seen from $h_{s c}$ evaluated at radius $r=0$ (dashed green line) and $r=a / 2$ (dotted red line). 


$$
\left|E_{z}\right|=\frac{e}{2 S \epsilon_{0}},
$$

so that the interaction causes an energy change per charge $e$, of

$$
h_{s c}(\zeta)=\frac{e^{2} L_{a}}{2 \epsilon_{0} S \gamma m_{e} c^{2}} \operatorname{sgn}(\zeta)=\frac{2 \pi r_{e} L_{a}}{S \gamma} \operatorname{sgn}(\zeta),
$$

with classical electron radius $r_{e} \equiv e^{2} / 4 \pi \epsilon_{0} m_{e} c^{2}$.

\section{B. Space charge Fourier transform}

In the simplified case of infinite sheets, the step function at $\zeta=0$ dominates $\tilde{h}(k)$, and we find a purely imaginary Fourier transform,

$$
\tilde{h}(k)=\frac{i A_{s c}}{k}
$$

with definition

$$
A_{s c} \equiv \frac{4 \pi r_{e} L_{a}}{S \gamma}
$$

Following Eq. (14), we then define the suppression parameter for space charge, $\Upsilon_{s c} \equiv n_{0} R_{56} A_{s c}$, and we expect broadband suppression for $Y_{s c}=1$.

In the finite sheet model, when $\zeta \gg a / \gamma$ the interaction falls off as $1 / \zeta^{2}$. The cutoff for $h(\zeta)$ as $\zeta \rightarrow \infty$ determines the noise suppression at low frequencies; the approximation of $\tilde{h}(k) \propto 1 / k$ breaks and we expect suppression to be frequency dependent for small $k$. Averaging the energy modulation across the disk gives (see e.g. $[16,17]^{1}$ )

$$
\tilde{h}(k)=\frac{i A_{s c}}{k}\left[1-2 I_{1}(k) K_{1}(k)\right]
$$

with modified Bessel functions $I_{1}(x), K_{1}(x)$. As $k \rightarrow \infty$, we find $\tilde{h}(k) \rightarrow i A_{s c} / k$, reproducing the result for the infinite sheet [Eq. (22)]. However, as $k \rightarrow 0$, we find $\tilde{h}(k) \rightarrow 0$, and we expect weaker noise suppression.

\section{Space charge simulation}

To check our analytical result, we simulate the interaction between particles in a 1D code. We load $N$ particles randomly within a bunch length $L$, with initial energy spread $\sigma_{\eta}$. A particle at location $z_{0}$ interacts with all particles within the range $z_{0}-L_{h}<z<z_{0}+L_{h}$, and we choose the interaction distance $L_{h}$ so that $L \gg L_{h} \gg a / \gamma$. To avoid edge effects from a finite bunch, we enforce periodic boundary conditions on the interaction. Following the interaction, the longitudinal positions shift according to $\hat{z}=z+R_{56} \hat{\eta}$, where the relative energy $\hat{\eta}$ is solely determined by the interactions of the first stage. We can then calculate the noise factor (or equivalently the fastFourier transform) of the resulting distribution, though

\footnotetext{
${ }^{1}$ In Eq. (2) of Ref. [17], the Bessel function should be $K_{1}$, not $K_{0}$.
}
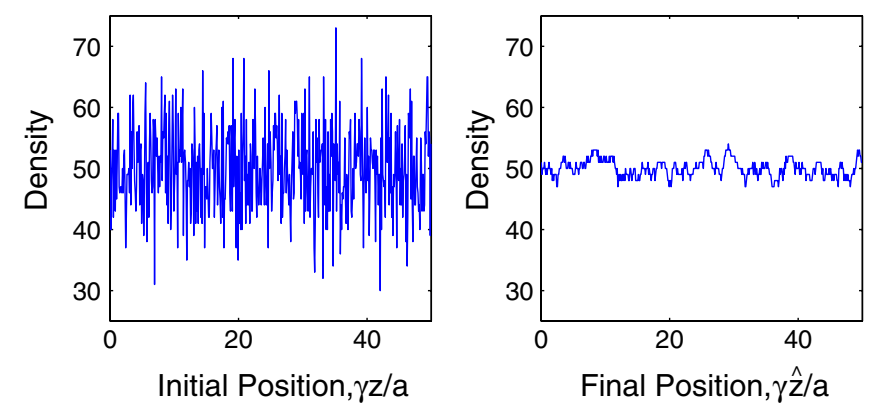

FIG. 4. On the left we show a histogram of particle density for particles loaded with random longitudinal positions. At right, following the interaction and dispersive regions, we find a reduction in noise in the equivalent histogram. (The example has $n_{0} a=5 \times 10^{2}, Y=1$.)

even by eye it is apparent we have suppressed high frequency noise (Fig. 4). In the limit of a cold beam, the 1D space charge interaction results in regularly spaced particles, each separated by the local inverse density $1 / n_{0}$ (Fig. 5).

We check the analytical solution [Eqs. (13) and (24)] against the simulations in Fig. 6. For all space charge simulations, units of length are normalized to the sheet radius $a$, and for now we assume zero initial energy spread $\sigma_{\eta}=0$.

\section{Validity of 1D model}

Throughout the paper we use a 1D model of sheet particles (sheets distributed with random longitudinal positions), so we would like to check that the resulting interaction, Eq. (24), is a reasonable approximation of a 3D distribution of particles. We may look to Ref. [15], which

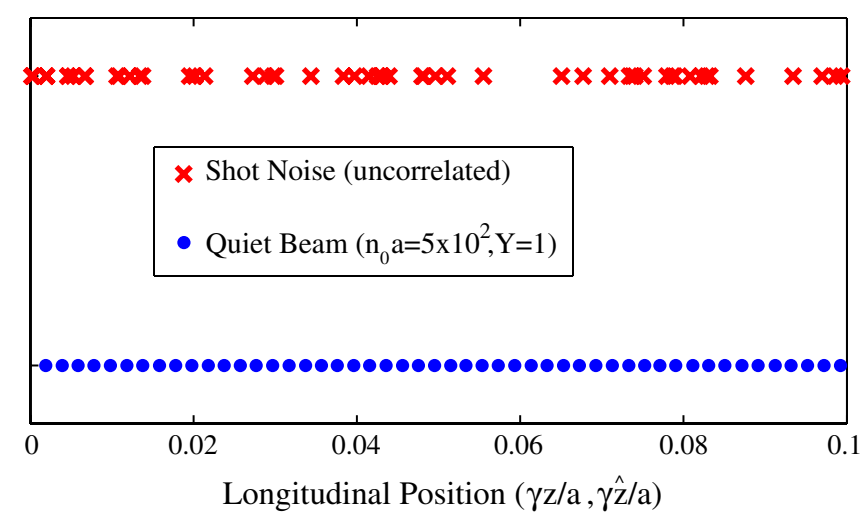

FIG. 5. Longitudinal distribution of particles in simulation before $(X)$ and after $(*)$ the noise suppression process. For $\sigma_{\eta} \ll 1 / R_{56} n_{0}$ and a $1 \mathrm{D}$ beam, it is possible to show that the initially uncorrelated distribution gives way to a regularly spaced beam with interparticle spacing $1 / n_{0}$ (see Appendix B). The regular structure amplifies bunching at very high frequencies $k=2 \pi n_{0}$ and its harmonics, while suppressing $F(k)$ at frequencies below $2 \pi n_{0}$. 


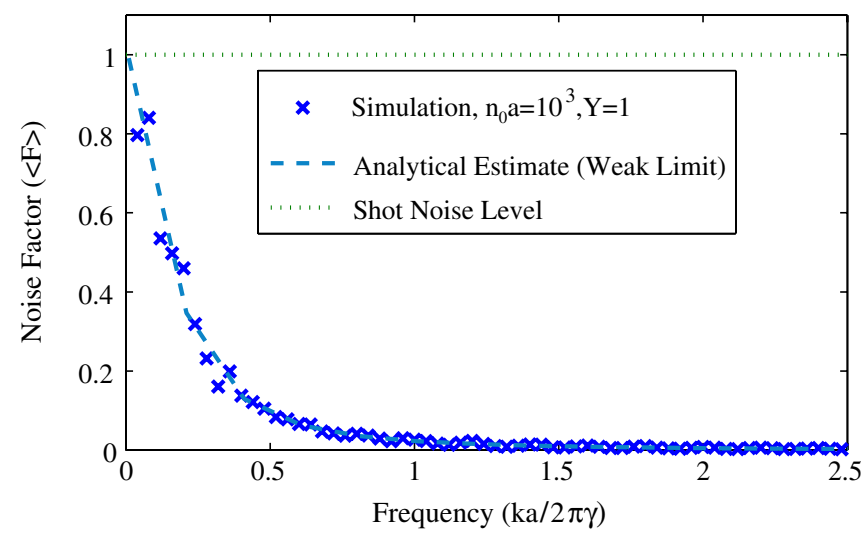

FIG. 6. A comparison of simulation and analytical results shows noise suppression as a function of frequency. With $Y \approx 1$ at high frequency, we find strong suppression. At low frequencies $(k \lesssim 2 \pi / a)$, we no longer have $\tilde{h}_{u}(k) \propto 1 / k$, so suppression is weaker for the given parameters.

studies the difference between 1D and 3D models of longitudinal space charge in the high frequency limit. Though the 1D and 3D distributions of longitudinal fields diverge at high frequency [see Eqs. (9),11-13 from Ref. [15] ], we find that when averaged transversely, the two models give approximately equal results.

The assumption of rigid 1D sheets may also overestimate the noise suppression. Past work on noise suppression resulting from plasma oscillations has found that 3D models lead to weaker noise suppression [10,11]. In our 1D model we assume a rigid sheet of charge that moves uniformly due to the average longitudinal field, whereas in reality each particle moves independently. To check the validity of our 1D model, we have written a 3D version of the space charge simulation. We confirm the existence of noise suppression for $Y=1$, but with somewhat weaker level of suppression. The 3D theory and simulations will be published elsewhere.

\section{UNDULATOR RADIATION CASE}

\section{A. Undulator radiation interaction}

As a second example, we consider the case of a beam traveling through an undulator. In the $1 \mathrm{D}$ limit, we can write down a simple, closed form solution for the interaction due to a helical undulator [18], providing a convenient system for studying noise suppression. For this reason, we neglect the space charge component in the following analysis, though we will see that in the absence of an amplifier [12] the space charge effect is generally dominant. We then find the undulator interaction (see Appendix A 1 for a derivation)

$$
h_{u}(\zeta)= \begin{cases}-A_{u}\left(1-\frac{\zeta}{N_{u} \lambda_{0}}\right) \cos k_{0} \zeta & 0<\zeta<N_{u} \lambda_{0} \\ 0 & \text { otherwise }\end{cases}
$$

with definition

$$
A_{u} \equiv 2 \pi \frac{e^{2} K^{2} N_{u} \lambda_{u}^{2}}{S \gamma^{3} m_{e} c^{2} \lambda_{0}}=4 \pi \frac{r_{e} L_{u}}{S \gamma} \frac{K^{2}}{1+K^{2}},
$$

undulator strength parameter $K$, length $L_{u}$, period $\lambda_{u}$, and resonant wavelength $\lambda_{0}$. This $1 \mathrm{D}$ expression is valid in the limit

$$
a \gg \frac{\gamma}{k \sqrt{1+K^{2}}},
$$

with $a$ the transverse beam size [see Eq. (A20)].

\section{B. Undulator Fourier transform}

From Eq. (13), noise suppression originates from the imaginary component of the Fourier transform. For the undulator case,

$$
\begin{aligned}
\tilde{h}_{u}(k) & \equiv \int_{-\infty}^{\infty} d \zeta e^{i k \zeta} h_{u}(\zeta) \\
& =-A_{u} N_{u} \lambda_{0} \int_{0}^{1} d \bar{\zeta}(1-\bar{\zeta}) \cos \alpha \bar{\zeta} e^{i m \alpha \bar{\zeta}}
\end{aligned}
$$

with definitions $\bar{\zeta} \equiv \zeta / N_{u} \lambda_{0}, \alpha \equiv 2 \pi N_{u}$, and $m \equiv k / k_{0}$. Integrating gives

$$
\begin{aligned}
\tilde{h}_{u}(k)= & -i A_{u} N_{u} \lambda_{0}\left[\frac{m}{\left(m^{2}-1\right) \alpha}\right. \\
& \left.-i \frac{\left(1+m^{2}\right)\left(1-e^{i m \alpha}\right)}{\left(m^{2}-1\right)^{2} \alpha^{2}}\right],
\end{aligned}
$$

with $N_{u}$ assumed to be an integer. At high frequencies $(m \gg 1)$, we neglect the second term, and find a purely imaginary FT:

$$
\tilde{h}_{u}(k) \approx-i \frac{A_{u}}{k} .
$$

As in the space charge case, we use Eq. (14) to define the suppression parameter $\Upsilon_{u}=-A_{u} n_{0} R_{56}$. In general, $\tilde{h}_{u}(k)$ is not purely imaginary, as stipulated in Eq. (13). However, at high frequencies, the undulator interaction looks like a step function [with the purely imaginary Fourier transform in Eq. (30)], and the physical picture in Fig. 2 applies here as well. Again, $Y_{u}$ has no $k$ dependence, so we expect broadband suppression.

At low frequencies, the approximation in Eq. (30) fails and the Fourier transform will be complex. If we take the limit of $m \rightarrow 1$, then from Eq. (29) we find

$$
\tilde{h}_{u}\left(k=k_{0}\right)=-A_{u} N_{u} \lambda_{0}\left(\frac{1}{4}+\frac{i}{4 \alpha}\right) \text {, }
$$

which is approximately real. We then find $\langle F(k)\rangle \sim 1+$ $\left|\tilde{h}_{u}\left(k_{0}\right)\right|^{2}$ and consequently expect bunching to increase at low frequencies. Note that $\left|\tilde{h}_{u}\left(k_{0}\right)\right|=\left(N_{u} \pi / 2\right) Y_{u}$, so for $N_{u} \gg 1$, we can expect an enhancement of $\sim N_{u}^{2} \pi^{2} / 4$ at the fundamental when $Y=1$.

It is interesting to note that at high frequencies, the undulator interaction is strictly weaker than space charge 
[Eq. (26) vs Eq. (23)]. Because the interactions have opposite sign, the undulator would only act to dampen the noise suppression from space charge.

\section{Undulator simulation}

To check our analytical result, we again run the simulation code but with the undulator interaction [Eq. (25)] instead of space charge. We load $N$ particles randomly within a length $L \gg N_{u} \lambda_{0}$, and for the undulator case a particle at location $z_{0}$ interacts with all particles within the range $z_{0}-N_{u} \lambda_{0}<z<z_{0}$.

The simulations confirm both the analytical solution [Eq. (13), valid for $\left.\Gamma_{2} \ll 1\right]$ and the numerical integral [Eq. (17)]. In all undulator simulations, we normalize units of length to the resonant wavelength $\lambda_{0}$, and we assume zero initial energy spread $\sigma_{\eta}=0$. (In Appendix C, we consider the effects of initial energy spread and energy modulation to the beam.)

\section{Undulator numerical integration}

While we already know the noise factor in the weakinteraction limit from Eq. (14), we would like to calculate $\Gamma_{2}$ explicitly to evaluate the numerical integral. For $Y \approx 1$, we find the weak interaction limit is equivalent to $n_{0} \gg$ $k^{2} L_{u}$. While the weak approximation is valid for many realistic examples, to facilitate simulations we use low particle numbers, where the approximation fails. For that reason, we use the numerical integration, Eq. (17), to check our simulations without the assumption of weak interaction.

Plugging the undulator interaction into Eq. (10) yields (see Appendix A 2)

$$
\begin{aligned}
\Gamma_{2}^{(u)}(\bar{\zeta})= & \frac{k^{2} R_{56}^{2}}{L} A_{u}^{2} N_{u} \lambda_{0}\left(\frac{1}{6}+\frac{1}{4 \alpha^{2}}+\frac{1}{12 \alpha^{3}}\{\alpha(1-\bar{\zeta})\right. \\
& \times\left[\alpha^{2}\left(\bar{\zeta}^{2}+\bar{\zeta}-2\right)-3\right] \cos (\alpha \bar{\zeta}) \\
& \left.\left.+3\left[\alpha^{2}(1-\bar{\zeta})-1\right] \sin (\alpha \bar{\zeta})\right\}\right) .
\end{aligned}
$$

Plugging into Eq. (16) gives constant term for the undulator interaction

$$
\bar{\Gamma}_{2}^{(u)}=\frac{n_{0} \alpha k_{0}\left(m R_{56} A_{u}\right)^{2}}{N}\left[\frac{1}{6}+\frac{1}{4 \alpha^{2}}\right]
$$

and then from Eq. (17) we find

$$
\begin{aligned}
\langle F(k)\rangle= & +2 n_{0} \alpha e^{-k^{2} R_{56}^{2} \sigma_{\eta}^{2}}\left[A_{u} m R_{56} \int_{0}^{1} d \bar{\zeta} e^{-N \Gamma_{2}^{(u)}(1-\bar{\zeta})}\right. \\
& \times \cos (\alpha \bar{\zeta}) \sin (m \alpha \bar{\zeta})+\frac{1}{k_{0}} \int_{0}^{1} d \bar{\zeta}\left(e^{-N \Gamma_{2}^{(u)}}\right. \\
& \left.\left.-e^{-N \bar{\Gamma}_{2}^{(u)}}\right) \cos (m \alpha \bar{\zeta})\right]
\end{aligned}
$$

which can be integrated directly. Simulations for the case of $N_{u}=1$ show good agreement with both the analytical result, Eqs. (13) and (29), and the numerical integration of Eq. (34), though as expected the analytical result fails for

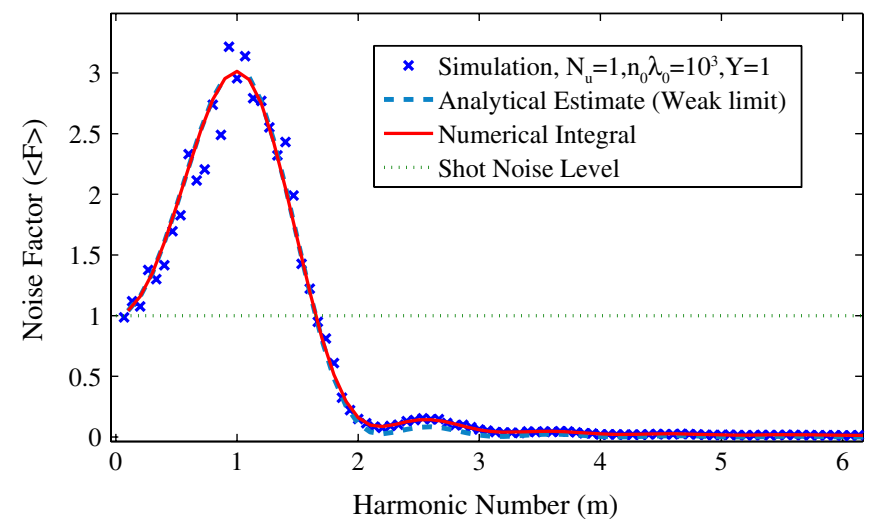

FIG. 7. A comparison of simulation, analytical result and numerical integral shows noise suppression at high frequency for $Y=1$. At low frequencies $(m \sim 1)$, we find $\tilde{h}_{u}(k)$ is approximately real [Eq. (31)], and bunching increases to $F(k) \approx$ $1-2 n_{0} k_{0} R_{56} \operatorname{Im}\left[\tilde{h}_{u}\left(k_{0}\right)\right]+\left(n_{0} k R_{56}\right)^{2}\left|\tilde{h}_{u}\left(k_{0}\right)\right|^{2} \approx 3$.

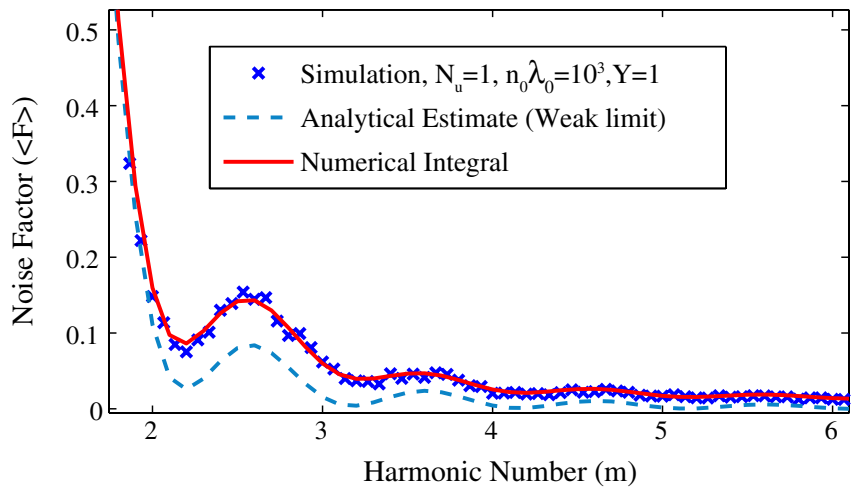

FIG. 8. A close-up of Fig. 7 shows agreement with the analytical expression starts to fail for $m \gtrsim 2$, but the numerical integral matches well everywhere.

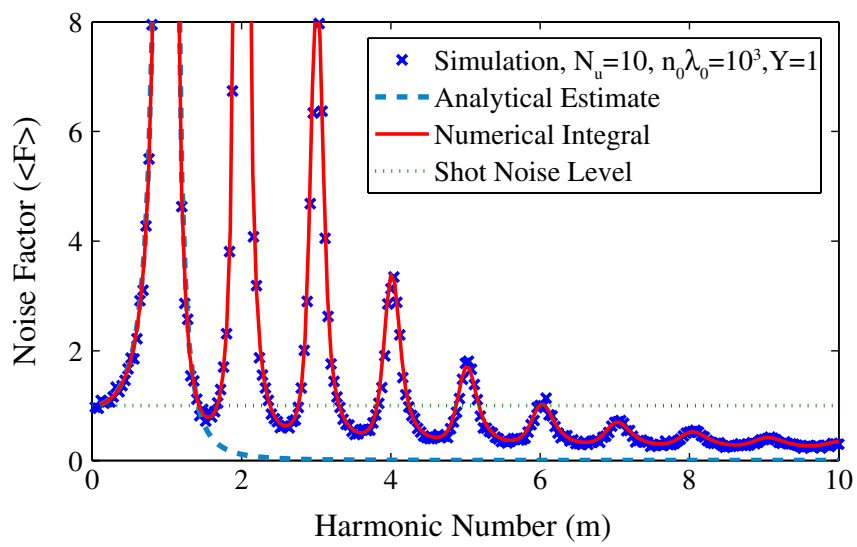

FIG. 9. A simulation example for ten undulator periods shows good agreement with the numerical integral. 


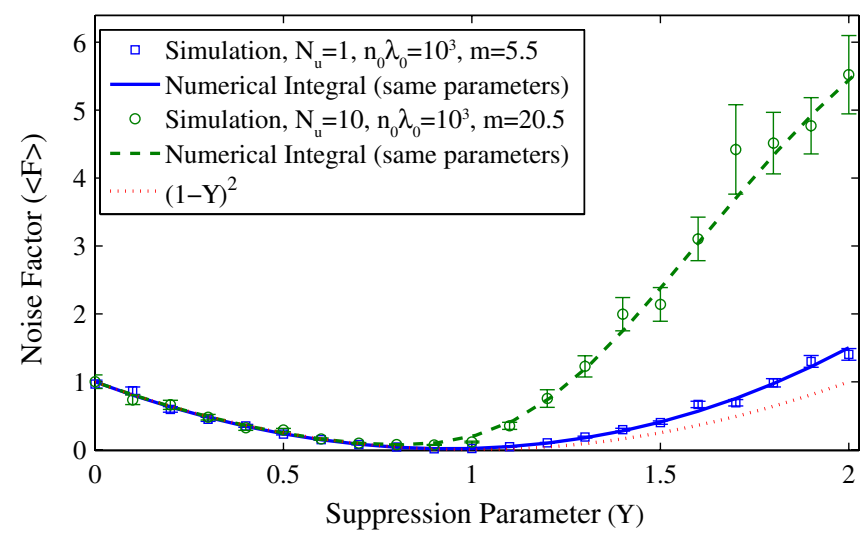

FIG. 10. Simulations show the noise factor for both $N_{u}=1$ and $N_{u}=10$ as a function of $Y$. When the approximation $k^{2} N_{u} \lambda_{0} / n_{0} \gg 1$ is valid, the noise scales as $(1-Y)^{2}$. We have chosen $m$ so that $\tilde{h}_{u}(k)$ is approximately imaginary.

$n_{0} \sim k^{2} L_{u}$ (Figs. 7 and 8). For a case with a longer undulator $\left(N_{u}=10\right)$, the numerical integration is essential for comparison with simulations (Fig. 9). Simulations confirm the noise factor's quadratic dependence on $Y$ [Eq. (14)] when in the weak interaction limit (Fig. 10). At this point we can also explicitly confirm the result from Sec. II C by plugging $\tilde{h}(k)$ and $\Gamma_{2}^{(u)}(\bar{\zeta})$ back into Eqs. (8) and (10) (see Appendix A 2).

\section{EXAMPLE PARAMETERS}

Though the focus of this paper is strictly theoretical, we calculate the interaction strength for SLAC's Next Linear Collider Test Facility to illustrate the scale of parameters involved. For the case of space charge over a length of $L_{a} \sim 10 \mathrm{~m}$ with beam cross section $S \sim 10^{-6} \mathrm{~m}^{2}$ and energy $\gamma \sim 100 \mathrm{MeV}$, we find $A_{s c} \equiv \frac{4 \pi r_{e} L_{a}}{S \gamma} \approx 2 \times 10^{-9}$. A beam of $20 \mathrm{~A}\left(n_{0}=4 \times 10^{11} \mathrm{~m}^{-1}\right)$ then needs $R_{56} \sim$ $2 \mathrm{~mm}$ to produce $Y=1$. We note that we are within the $1 \mathrm{D}$ limit even for optical wavelengths $\left(k_{0} \sigma / \gamma \gtrsim 25\right)$.

For the undulator radiation to dominate over the space charge interaction, we may use an amplifier, as proposed by Litvinenko [12]. The increase in the interaction strength also has the benefit of decreasing the required dispersion $R_{56}$, allowing for larger energy spreads and higher frequency suppression. However, the larger modulation may increase the beam energy spread (see Appendix C).

\section{CONCLUSION}

We present a longitudinal 1D model of shot noise suppression for a simplified system of an interaction region followed by a dispersive region. In the limit of small energy spread $\left(\left|k R_{56} \sigma_{\eta}\right| \ll 1\right)$, interactions with primarily imaginary Fourier transforms can suppress the noise factor below the shot noise level. We work out the specific cases of undulator and space charge interactions, and confirm both results with a $1 \mathrm{D}$ simulation. We note that a wide range of imaginary impedances (e.g., linac wakefields) may also reduce shot noise. In the 1D limit with small energy spread, the suppression process may amplify bunching at very high frequencies near the interparticle spacing $1 / n_{0}$.

\section{ACKNOWLEDGMENTS}

We would like to thank K. J. Kim and R. Lindberg for helpful discussions. This work was supported by U.S. DOE Contract No. DE-AC02-76SF00515.

\section{APPENDIX A: DERIVATIONS FOR NOISE SUPPRESSION FOR THE UNDULATOR INTERACTION}

\section{Helical undulator interaction in the 1D limit}

In this Appendix we will derive Eq. (25) for the interaction of two slices of a bunch separated by distance $\zeta$ during passage through a helical undulator and obtain an applicability condition for our 1D approximation. For now, we only consider the transverse field $E_{\perp}$; the contribution from the longitudinal space charge field $E_{z}$ is given in Sec. III, with $\gamma$ replaced by $\gamma_{z}=\gamma / \sqrt{1+K^{2} / 2}$ due to the presence of the undulator [19].

Our derivation is based on the paraxial approximation for the field of a relativistic particle from Ref. [20]. The Fourier component (indicated by hat) of the field of a point charge $q$ (which we call a source charge) at a point with coordinates $x, y, z$ is given by the following formula [see Eq. (28) in [20] ]:

$\hat{\vec{E}}_{\perp}(x, y, z, \omega)=\frac{i \omega q}{c^{3}} \int_{-\infty}^{\infty} \frac{d z^{\prime}}{z-z^{\prime}} H\left(z-z^{\prime}\right) e^{i \Phi}\left[\vec{v}_{\perp}\left(z^{\prime}\right)+\vec{a}\left(z^{\prime}\right)\right]$,

where

$$
\begin{aligned}
\Phi= & \omega\left[\frac{s\left(z^{\prime}\right)}{v}-\frac{z^{\prime}}{c}+\frac{z}{c}\right]+\frac{\omega}{2 c\left(z-z^{\prime}\right)}\left\{\left[x-x_{0}\left(z^{\prime}\right)\right]^{2}\right. \\
& \left.+\left[y-y_{0}\left(z^{\prime}\right)\right]^{2}\right\},
\end{aligned}
$$

and $H$ is the step function. In these equations $\vec{v}_{\perp}(z)$ is the transverse component of the particle's velocity as a function of coordinate $z, x_{0}(z)$ and $y_{0}(z)$ define the particle trajectory, $s(z)$ is the length of the trajectory as a function of $z$, and $v$ is the absolute value of particle velocity that is assumed constant. The vector $\vec{a}\left(z^{\prime}\right)$ is

$$
\vec{a}\left(z^{\prime}\right)=-c \frac{x-x_{0}\left(z^{\prime}\right)}{z-z^{\prime}} \vec{x}-c \frac{y-y_{0}\left(z^{\prime}\right)}{z-z^{\prime}} \vec{y},
$$

with $\vec{x}$ and $\vec{y}$ the unit vectors in corresponding directions. The step function under the integral [Eq. (A1)] is missing in Eq. (28) of [20] - a mistake that was corrected by the authors in a later publication [19].

Let us consider a helical undulator of length $L_{u}$. Inside the undulator, $0<z<L_{u}$, the transverse velocity of the particle and its orbit are 


$$
\begin{gathered}
\vec{v}_{\perp}(z)=\frac{c K}{\gamma}\left(\vec{x} \sin k_{u} z+\vec{y} \cos k_{u} z\right), \quad x_{0}(z)=-\frac{K}{\gamma k_{u}} \cos k_{u} z, \\
y_{0}(z)=\frac{K}{\gamma k_{u}} \sin k_{u} z,
\end{gathered}
$$

where $K$ is the undulator parameter, $\gamma$ is the Lorentz factor, and $k_{u}=2 \pi / \lambda_{u}$ with $\lambda_{u}$ the undulator period. Note that since the transverse velocity of the source charge is zero outside of the undulator, the integration over $z^{\prime}$ in Eq. (A1) is actually limited to the interval $0<z^{\prime}<L_{u}$.

Let us now consider a test particle of charge $e$ traveling in front of the source particle on a parallel trajectory shifted in the transverse direction by vector $X \vec{x}+Y \vec{y}$ in such a way that it passes through each point $z$ earlier than the source particle by time $T>0$. The current density associated with the test particle is

$$
\begin{aligned}
\vec{j}(x, y, z, t)= & \frac{e}{v_{z}} \vec{v}(z) \delta\left[x-x_{0}(z)-X\right] \delta\left[y-y_{0}(z)-Y\right] \\
& \times \delta\left(\frac{s(z)}{v}-t-T\right) .
\end{aligned}
$$

We can calculate the energy change $U$ of the test particle due to the interaction with the source one as the product $\vec{E} \cdot \vec{j}$ integrated over the space and time

$U=\int_{-\infty}^{\infty} d t \int_{-\infty}^{\infty} d x \int_{-\infty}^{\infty} d y \int_{0}^{L_{u}} d z \vec{E}_{\perp}(x, y, z, t) \cdot \vec{j}(x, y, z, t)$,

where we limited integration over $z$ from 0 to $L_{u}$ taking into account that $\vec{E} \cdot \vec{j}=0$ outside of the undulator, where the transverse component of the test particle velocity is equal to zero. Substituting Eq. (A5) into Eq. (A6) and expressing the field $\vec{E}_{\perp}(x, y, z, t)$ through its Fourier transform $\vec{E}_{\perp}(x, y, z, t)=(2 \pi)^{-1} \int d \omega e^{i \omega t} \hat{\vec{E}}_{\perp}(x, y, z, \omega)$ using Eq. (A1), one finds

$$
\begin{aligned}
U= & e \int_{0}^{L_{u}} \vec{E}_{\perp}\left(x_{0}(z)+X, y_{0}(z)+Y, z, \frac{s(z)}{v}-T\right) \cdot \vec{v}_{\perp}(z) \frac{d z}{v_{z}(z)} \\
= & \frac{e}{2 \pi c} \int_{-\infty}^{\infty} d \omega \int_{0}^{L_{u}} e^{i \omega[T-s(z) / v]} \hat{\vec{E}}_{\perp}\left[x_{0}(z)+X, y_{0}(z)\right. \\
& +Y, z, \omega] \cdot \vec{v}_{\perp}(z) d z \\
= & \frac{i e q}{2 \pi c^{4}} \int_{-\infty}^{\infty} \omega d \omega \int_{0}^{L_{u}} \frac{d z d z^{\prime}}{z-z^{\prime}} H\left(z-z^{\prime}\right) \\
& \times\left[\vec{v}_{\perp}\left(z^{\prime}\right)+\vec{a}\left(z^{\prime}\right)\right] \cdot \vec{v}_{\perp}(z) e^{i \Phi_{1}},
\end{aligned}
$$

with

$$
\begin{aligned}
\Phi_{1}= & \omega\left[\frac{s\left(z^{\prime}\right)}{v}-\frac{z^{\prime}}{c}-\frac{s(z)}{v}+\frac{z}{c}+T\right]+\frac{\omega}{2 c\left(z-z^{\prime}\right)}\left\{\left[X+x_{0}(z)\right.\right. \\
& \left.\left.-x_{0}\left(z^{\prime}\right)\right]^{2}+\left[Y+y_{0}(z)-y_{0}\left(z^{\prime}\right)\right]^{2}\right\},
\end{aligned}
$$

where we approximated $v_{z} \approx c$.

Let us now assume that instead of a source point charge $q$ we are dealing with a thin uniformly charged disk of radius $a$ moving in the undulator according to Eq. (A4). This disk represents a thin slice of an electron bunch. We assume that the radius $a$ is much larger than the orbit deviations from the straight line, $a \gg\left|x_{0}(z)\right|,\left|y_{0}(z)\right|$. To calculate the energy change $U$ of the test charge due to the interaction with the source disk $q$, we need to average Eq. (A7) over the disk surface, that is to calculate

$$
\bar{U}=\frac{1}{S} \int_{S} U d X d Y,
$$

where $S$ is the cross section area of the disk and the subscript $S$ at the integral sign indicates integration of the surface of the disk. Note that this integration cancels the term involving $\vec{a}\left(z^{\prime}\right)$ in Eq. (A7), because it is an antisymmetric function of $X$ and $Y$ [in the limit $X \gg\left|x_{0}(z)\right|$ and $\left.Y \gg\left|y_{0}(z)\right|\right]$. Calculation of $\bar{U}$ involves the following integral:

$$
\begin{aligned}
I= & \int_{S} d X d Y \exp \left[\frac { \omega } { 2 c ( z - z ^ { \prime } ) } \left\{\left[X+x_{0}(z)-x_{0}\left(z^{\prime}\right)\right]^{2}\right.\right. \\
& \left.\left.+\left[Y+y_{0}(z)-y_{0}\left(z^{\prime}\right)\right]^{2}\right\}\right] .
\end{aligned}
$$

If the factor $\omega / 2 c\left(z-z^{\prime}\right)$ can be considered as large, that is

$$
\left|\frac{\omega a^{2}}{2 c\left(z-z^{\prime}\right)}\right| \gg 1,
$$

integration over $X$ and $Y$ can be extended from $-\infty$ to $\infty$ with the result

$$
I=\frac{2 i \pi c\left(z-z^{\prime}\right)}{\omega} .
$$

We assume that this is the case, and postpone discussion of the condition Eq. (A11) toward the end of this section. We then have

$$
\begin{aligned}
\bar{U}= & -\frac{e q}{S c^{3}} \int_{-\infty}^{\infty} d \omega \int_{0}^{L_{u}} d z d z^{\prime} H\left(z-z^{\prime}\right) \vec{v}_{\perp}\left(z^{\prime}\right) \cdot \vec{v}_{\perp}(z) \\
& \times \exp \left(i \omega\left[\frac{s\left(z^{\prime}\right)}{v}-\frac{z^{\prime}}{c}-\frac{s(z)}{v}+\frac{z}{c}+T\right]\right) .
\end{aligned}
$$

Using $\gamma \gg 1$, it is easy to find that in the helical undulator

$$
\frac{1}{v} s(z) \approx \frac{z}{c}+\frac{z}{2 c \gamma^{2}}\left(1+K^{2}\right),
$$

and

$$
\vec{v}_{\perp}\left(z^{\prime}\right) \cdot \vec{v}_{\perp}(z)=\frac{c^{2} K^{2}}{\gamma^{2}} \cos k_{u}\left(z-z^{\prime}\right),
$$

which gives 


$$
\begin{aligned}
\bar{U}= & -\frac{e q K^{2}}{S c \gamma^{2}} \int_{-\infty}^{\infty} d \omega \int_{0}^{L_{u}} d z d z^{\prime} H\left(z-z^{\prime}\right) \cos k_{u}\left(z-z^{\prime}\right) \\
& \times \exp \left(-i \omega\left[\frac{z-z^{\prime}}{2 c \gamma^{2}}\left(1+K^{2}\right)-T\right]\right) \\
= & -\frac{2 \pi e q K^{2}}{S c \gamma^{2}} \int_{0}^{L_{u}} d z \int_{0}^{z} d z^{\prime} \cos k_{u}\left(z-z^{\prime}\right) \\
& \times \delta\left(\frac{z-z^{\prime}}{2 c \gamma^{2}}\left(1+K^{2}\right)-T\right) .
\end{aligned}
$$

Noting that the integral over the $\delta$ function can be written in terms of step functions,

$$
\begin{aligned}
& \int_{0}^{z} d z^{\prime} \delta\left(\frac{z-z^{\prime}}{2 c \gamma^{2}}\left(1+K^{2}\right)-T\right) \\
& =\frac{2 c \gamma^{2}}{1+K^{2}} H(T) H\left(\frac{z}{2 c \gamma^{2}}\left(1+K^{2}\right)-T\right),
\end{aligned}
$$

we calculate the last integral in Eq. (A16) as

$$
\bar{U}=-\frac{4 \pi e q L_{u}}{S} \frac{K^{2}}{1+K^{2}}\left(1-\frac{2 c \gamma^{2} T}{L_{u}\left(1+K^{2}\right)}\right) \cos \frac{2 c \gamma^{2} k_{u} T}{1+K^{2}} .
$$

Observing that $\zeta=c T, \quad \lambda_{0}=\lambda_{u}\left(1+K^{2}\right) / 2 c \gamma^{2}$, and $h=\bar{U} / \gamma m c^{2}$, it is easy to see that Eq. (A18) coincides with Eqs. (25) and (26).

Let us now discuss the applicability condition Eq. (A11). The frequency $\omega$ in this inequality we can estimate as $T^{-1}$, and characteristic value of $z-z^{\prime}$ is $2 c \gamma^{2} T /\left(1+K^{2}\right)$, which gives

$$
\left|\frac{\omega a^{2}}{2 c\left(z-z^{\prime}\right)}\right| \sim \frac{a^{2}\left(1+K^{2}\right)}{c^{2} \gamma^{2} T^{2}}=\frac{a^{2}\left(1+K^{2}\right)}{\gamma^{2} \zeta^{2}} \gg 1 .
$$

Equivalently, since we use the Fourier transformation of the function $h$ [see Eq. (28)], with $k=\omega / c$, we can write the applicability condition as

$$
\frac{k^{2} a^{2}\left(1+K^{2}\right)}{\gamma^{2}} \gg 1
$$

\section{Quadratic term $\left(\boldsymbol{\Gamma}_{2}\right)$ for helical undulator}

We calculate $\Gamma_{2}$ explicitly for use in the numerical integral [Eq. (34)]. Plugging Eq. (25) into Eq. (10) gives

$$
\begin{aligned}
\Gamma_{2}(\zeta)= & n_{0} k^{2} R_{56}^{2}\left[\int_{-N_{u} \lambda_{0}}^{0} d \tau h_{u}(-\tau)^{2}\right. \\
& \left.-\int_{-N_{u} \lambda_{0}+\zeta}^{0} d \tau h_{u}(-\tau+\zeta) h_{u}(-\tau)\right],
\end{aligned}
$$

where we have used $h_{u}(x)=0$ outside of the range $0<x<N_{u} \lambda_{0}$ to choose the integration limits. Plugging in for $h_{u}$, defining normalized variables $\bar{\tau} \equiv \tau / N_{u} \lambda_{0}$, $\bar{\zeta} \equiv \zeta / N_{u} \lambda_{0}$, and $\alpha \equiv 2 \pi N_{u}$, we have

$$
\begin{aligned}
\Gamma_{2}(\bar{\zeta})= & n_{0} k^{2} R_{56}^{2} A_{u}^{2} N_{u} \lambda_{0}\left[\int_{-1}^{0} d \bar{\tau}(1+\bar{\tau})^{2} \cos (\alpha \bar{\tau})^{2}\right. \\
& -\int_{\bar{\zeta}-1}^{0} d \bar{\tau}(1+\bar{\tau}-\bar{\zeta})(1+\bar{\tau}) \\
& \times \cos [\alpha(\bar{\tau}-\bar{\zeta})] \cos (\alpha \bar{\tau})] .
\end{aligned}
$$

We can then integrate to find

$$
\begin{aligned}
\Gamma_{2}(\bar{\zeta})= & n_{0} k^{2} R_{56}^{2} A_{u}^{2} N_{u} \lambda_{0}\left[\frac{1}{6}+\frac{1}{4 \alpha^{2}}\right. \\
& +\frac{1}{12 \alpha^{3}}\left\{\alpha(1-\bar{\zeta})\left[\alpha^{2}\left(\bar{\zeta}^{2}+\bar{\zeta}-2\right)-3\right] \cos (\alpha \bar{\zeta})\right. \\
& \left.\left.+3\left[\alpha^{2}(1-\bar{\zeta})-1\right] \sin (\alpha \bar{\zeta})\right\}\right],
\end{aligned}
$$

where we have assumed an integer number of undulator periods $N_{u}$ to simplify the trigonometric functions. Note that this expression is valid only for $0<\bar{\zeta}<1$; while Eq. (A23) does not look symmetric about $\zeta=0$, from Eq. (10) we know that $\Gamma_{2}$ is an even function of $\bar{\zeta}$.

In the main text, we use $\Gamma_{2}$ to evaluate Eq. (17). We can also use $\Gamma_{2}$ to explicitly confirm the result from Sec. IIC for the high frequency undulator case by plugging Eq. (A23) into Eq. (11). With $\Gamma_{2}$ an even function, we can write the third (quadratic) term from Eq. (11) as

$$
\left\langle F_{2}(k)\right\rangle \equiv 2 n_{0} e^{-k^{2} R_{56}^{2} \sigma_{\eta}^{2}} N_{u}^{2} \lambda_{0}^{2} \int_{0}^{L} d \bar{\zeta} \cos (m \alpha \bar{\zeta}) N \Gamma_{2}(\bar{\zeta}) .
$$

We are interested in $k \neq 0$, so as in Eq. (17), we subtract off the two constant terms. By definition, the product of interactions must disappear for $\bar{\zeta}>1$ (when at least one of $z_{1}$ and $z_{2}$ cannot interact with the test charge), so we set the upper limit to 1 and integrate to find

$$
\begin{aligned}
\left\langle F_{2}(k)\right\rangle= & -2 n_{0}^{2} k^{2} R_{56}^{2} A_{u}^{2} N_{u} \lambda_{0} e^{-k^{2} R_{56}^{2} \sigma_{\eta}^{2}} \frac{1}{4 \alpha^{4}\left(m^{2}-1\right)^{4}} \\
& \times\left\{2 m^{2} \alpha^{2}\left(m^{2}-1\right)^{2}+4\left(m^{2}+1\right)^{2}[\cos (m \alpha)-1]\right. \\
& \left.+4 m \alpha\left(m^{4}-1\right) \sin (m \alpha)\right\} .
\end{aligned}
$$

In the limit $m \gg 1$ the result simplifies to

$$
\left\langle F_{2}(k)\right\rangle=n_{0}^{2} k^{2} R_{56}^{2} A_{u}^{2} N_{u}^{2} \lambda_{0}^{2} e^{-k^{2} R_{56}^{2} \sigma_{\eta}^{2}} \frac{1}{m^{2} \alpha^{2}}=\Upsilon_{u}^{2} e^{-k^{2} R_{56}^{2} \sigma_{\eta}^{2}} .
$$

Adding in the first two terms of Eq. (13) and taking the limit of $m \gg 1$ and $\sigma_{\eta}=0$, we confirm $\langle F(k)\rangle=\left(1-\Upsilon_{u}\right)^{2}$.

\section{APPENDIX B: BUNCHING AT THE AVERAGE INTERPARTICLE SPACING}

In the cold, sheet-beam limit (treating each particle as a sheet), we show that an interaction $h(\zeta)$ results in nearly full bunching factor at the intersheet spacing if there is a step function at $\zeta=0$ (Fig. 5). To facilitate the calculation, 
we number our particles (sheets) such that $z_{i}<z_{k}$ if $i<k$. The energy change for particles $k$ and $k+1$ is given by

$$
\Delta E_{k}=\sum_{i=1}^{N} h\left(z_{k}-z_{i}\right), \quad \Delta E_{k+1}=\sum_{i=1}^{N} h\left(z_{k+1}-z_{i}\right)
$$

To find the difference in energy modulations, $\Delta E_{k+1}-\Delta E_{k}$, we rewrite $\Delta E_{k+1}$ in terms of $\Delta E_{k}$,

$$
\begin{aligned}
\Delta E_{k+1} & =h\left(z_{k+1}-z_{k}\right)+\sum_{i \neq k}^{N} h\left(z_{k+1}-z_{k}+z_{k}-z_{i}\right) \\
& \approx h\left(\Delta z_{k}\right)+\sum_{i \neq k}^{N} h\left(z_{k}-z_{i}\right)+\Delta z_{k} \sum_{i \neq k}^{N} h^{\prime}\left(z_{k}-z_{i}\right) \\
& =\Delta E_{k}+\left[h\left(\Delta z_{k}\right)-h\left(-\Delta z_{k}\right)\right]+\Delta z_{k} \sum_{i \neq k}^{N} h^{\prime}\left(z_{k}-z_{i}\right),
\end{aligned}
$$

where we have defined the initial distance between particles, $\Delta z_{k}=z_{k+1}-z_{k}$, and assumed that the average spacing $\langle\Delta z\rangle$ is small to make the Taylor expansion in the second step. \{Specifically, we assume $[h(\zeta+\Delta)-$ $h(\zeta)] / h(\zeta) \ll 1$, everywhere except at the step function. $\}$ Hence, the energy difference between the two neighboring particles is

$$
\Delta E_{k+1}-\Delta E_{k}=\left[h\left(\Delta z_{k}\right)-h\left(-\Delta z_{k}\right)\right]+\Delta z_{k} \sum_{i \neq k}^{N} h^{\prime}\left(z_{k}-z_{i}\right) .
$$

To compute the last sum, we replace the summation by an integration (assuming, as before, a uniform longitudinal distribution of particles in the beam). Skipping over the region where we have explicitly assumed there are no particles, for a longitudinally uniform beam we find

$$
\begin{aligned}
\sum_{i=1}^{N} h^{\prime}\left(z_{k}-z_{i}\right) & \approx n_{0}\left[\int_{-\infty}^{z_{k}^{-}} d z h^{\prime}\left(z_{k}-z\right)+\int_{z_{k+1}^{+}}^{\infty} d z h^{\prime}\left(z_{k}-z\right)\right] \\
& =n_{0}\left[\int_{\infty}^{0^{+}}(-d \zeta) h^{\prime}(\zeta)+\int_{-\Delta z_{k}^{+}}^{-\infty}(-d \zeta) h^{\prime}(\zeta)\right] \\
& \approx-n_{0}\left[h\left(0^{+}\right)-h\left(0^{-}\right)\right],
\end{aligned}
$$

where we have approximated $h\left(\Delta z_{k}^{ \pm}\right) \approx h\left(0^{ \pm}\right)$. For an interaction with a step function at $\zeta=0$ of amplitude $A=$ $\left[h\left(0^{+}\right)-h\left(0^{-}\right)\right]$, we can rewrite

$$
\Delta E_{k+1}-\Delta E_{k}=A\left(1-n_{0} \Delta z_{k}\right) .
$$

The energy difference $\Delta E_{k+1}-\Delta E_{k}$ depends linearly on the initial distance between the particles (Fig. 11).

Following the dispersive section of strength $R_{56}$, the new distance between particles $k$ and $k+1$ is

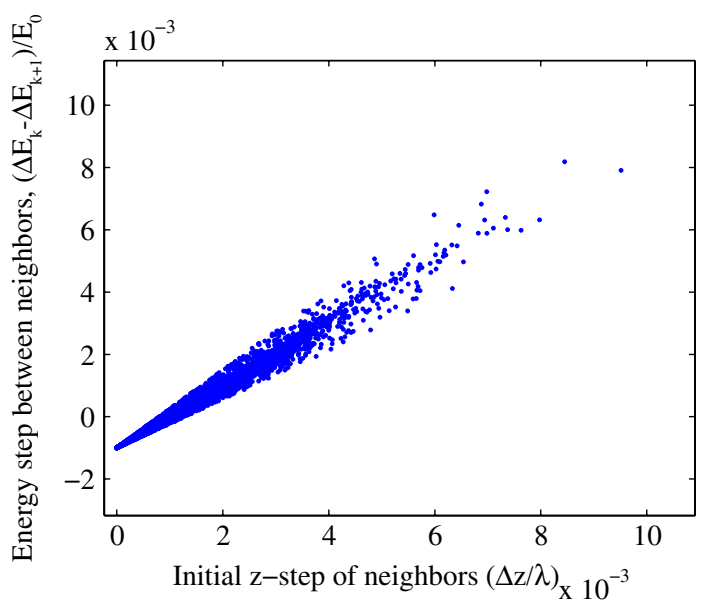

FIG. 11. The relative modulation between neighboring particles, $E_{k+1}-E_{k}$, is proportional to the initial distance between the particles (sheets), $\Delta z$. Simulation is for the undulator interaction, with $Y=1, n_{0} \lambda_{0}=10^{3}$. Particles that are closer (farther) than the interparticle spacing, $\Delta z<1 / n_{0}$, lose (gain) energy relative to the previous particle, and move away (closer) in negative dispersion.

$$
\begin{aligned}
\Delta z_{k}^{\text {new }} & =\Delta z_{k}+R_{56}\left(\Delta E_{k+1}-\Delta E_{k}\right) \\
& =R_{56} A+\left(1-R_{56} n_{0} A\right) \Delta z_{k} .
\end{aligned}
$$

At full suppression $Y=R_{56} n_{0} A=1$, we obtain

$$
\Delta z_{k}^{\text {new }}=\frac{1}{n_{0}},
$$

giving a uniform structure with interparticle spacing of $1 / n_{0}$, as in a quasicrystalline beam [21].

The increase in noise at low frequencies [Eq. (31)] leads to variation in local density, and thus variation in particle spacing. From simulations we confirm $\Delta z_{k}^{\text {new }}=1 / \bar{n}_{0}$, where $\bar{n}_{0}$ is the local density over a region of length $L_{n}$ with $1 / n_{0} \ll L_{n} \ll \lambda_{0} / N_{u}$. In reality, the beam is not transversely uniform as assumed in the 1D sheet model, so we do not expect a rigid quasicrystalline structure. However, we still expect to find an amplification of the bunching factor at the inverse of the interparticle spacing. Though the uniform beam is an intriguing theoretical result, due to the requirement of cold beam $\left(\sigma_{\eta} \ll\right.$ $\left.1 / n_{0} R_{56}\right)$ and a true step-function interaction, practical applications may prove elusive.

\section{APPENDIX C: EFFECTS OF ENERGY SPREAD AND MODULATION}

In the main text we have treated only the case of vanishing energy spread, $\sigma_{\eta} \rightarrow 0$. When $k R_{56} \sigma_{\eta} \geq 1$, the energy spread factor $\exp \left[-k^{2} R_{56}^{2} \sigma_{\eta}^{2}\right]$ washes out noise suppression; at high enough frequencies, noise suppression fails. To suppress noise for larger $k$ we must decrease the dispersive strength $R_{56}$. However, from $\Upsilon \equiv R_{56} n_{0} A=1$, 


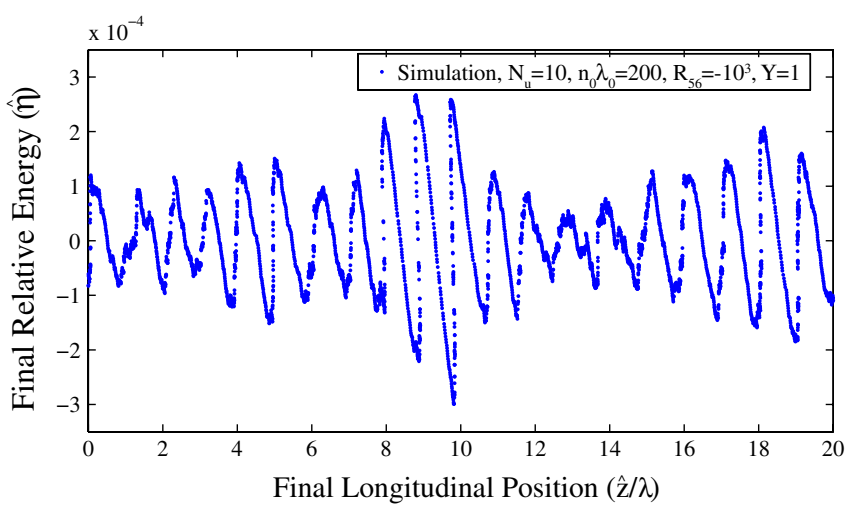

FIG. 12. Phase space of the particles following both the interaction and dispersive regions. The example is for the undulator case with uniform initial energy $\sigma_{\eta}=0$.

weak dispersion implies a strong interaction $A$, which in turn heats the beam. To determine an upper limit on the interaction strength, we consider how the energy modulation in the suppression process affects the energy spread of the beam.

\section{Energy modulation from noise suppression}

The noise suppression process (interaction and dispersive regions) changes the particle energies, $\eta \rightarrow \hat{\eta}$ (Fig. 12). For the undulator case, the expected amplitude of the resulting energy modulation for a single particle is

$$
\begin{aligned}
\left\langle h_{u}^{2}(\zeta)\right\rangle & =\int_{0}^{N_{u} \lambda_{0}} \frac{d \zeta}{N_{u} \lambda_{0}} h_{u}^{2}(\zeta)=A_{u}^{2} \int_{0}^{1} d \bar{\zeta}(1-\bar{\zeta})^{2} \cos ^{2} \alpha \bar{\zeta} \\
& =\frac{A_{u}^{2}}{24}\left(4+\frac{6}{\alpha^{2}}-\frac{2 \sin 2 \alpha}{\alpha^{3}}\right) .
\end{aligned}
$$

With $\alpha \equiv 2 \pi N_{u} \geq 2 \pi$, we drop the final two terms. We can then guess that if a particle interacts with on average

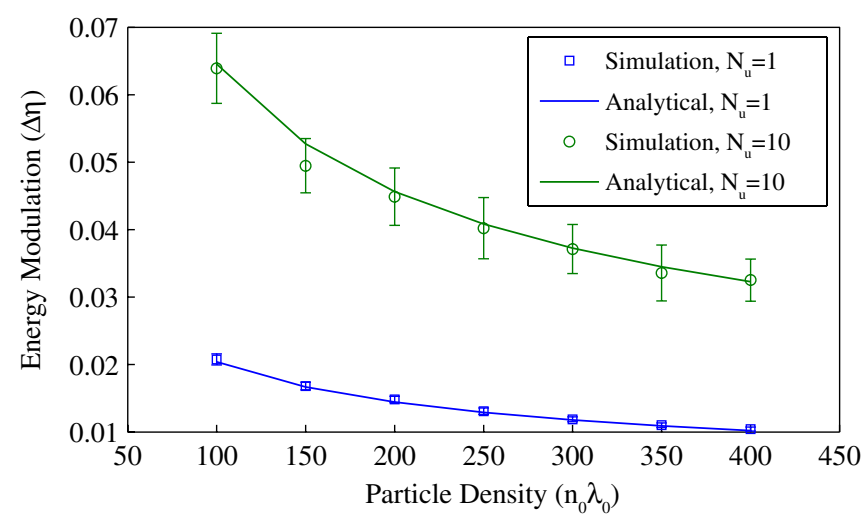

FIG. 13. Energy modulation $\langle\Delta \eta\rangle$ induced by the interaction [Eq. (C3)] is compared to the result of repeated simulations for the undulator case. The final energy spread is given as a function of the particle per wavelength $n_{0} \lambda_{0}$ for $N_{u}=1$ and 10 , and initial energy spread $\sigma_{\eta}=0$.

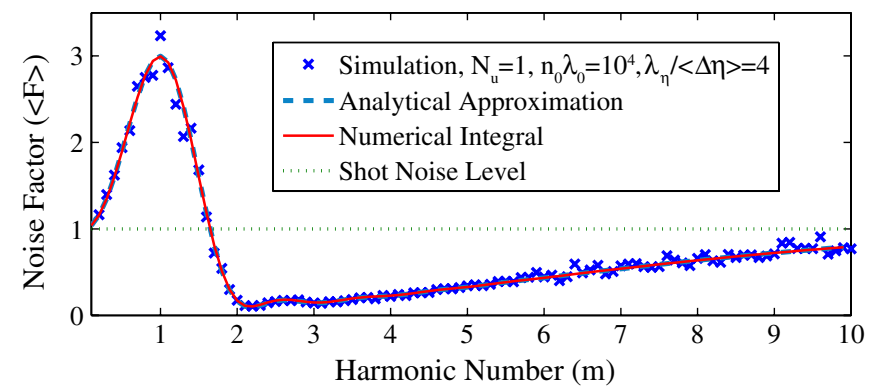

FIG. 14. Noise suppression for the case $N_{u}=1$ with $\langle\Delta \eta\rangle \approx$ $\sigma_{\eta} / 5$. For the parameters given, $\lambda_{\min } \approx \lambda_{0} / 8$. With higher charge density $n_{0}$, suppression extends to shorter wavelengths.

$\bar{N}=n_{0} N_{u} \lambda_{0}$ particles, the expected energy spread will be approximately

$$
\langle\Delta \eta\rangle \approx \sqrt{\bar{N}\left\langle h_{u}^{2}(\zeta)\right\rangle} \approx \sqrt{\frac{n_{0} N_{u} \lambda_{0}}{6}} A_{u} .
$$

Combined with the suppression condition $Y=$ $-n_{0} R_{56} A_{u} \sim 1$, we have

$$
\langle\Delta \eta\rangle \approx-\sqrt{\frac{N_{u} \lambda_{0}}{6 n_{0}}} \frac{1}{R_{56}},
$$

for the case of maximum suppression (Fig. 13).

\section{Suppression wavelength limit}

The energy spread washes out noise suppression for wavelengths below $\lambda_{\min } \sim 2 \pi \sigma_{\eta} R_{56}$. Expressing $\lambda_{\min }$ in terms of the energy modulation, we find

$$
\lambda_{\min }=2 \pi \sqrt{\frac{N_{u} \lambda_{0}}{6 n_{0}}} \frac{\sigma_{\eta}}{\langle\Delta \eta\rangle} .
$$

From Eq. (C4), we note that suppression is possible even when the modulation amplitude is small compared to the beam's natural energy spread, $\langle\Delta \eta\rangle \ll \sigma_{\eta}$ (Fig. 14). Decreasing $R_{56}$ extends suppression to shorter wavelengths, but heats the beam. If we require that the interaction has a negligible effect on the energy spread, then we find a lower limit on $\lambda_{\min }$ when $\langle\Delta \eta\rangle \sim \sigma_{\eta}$.

\section{Energy spread for FEL}

Our goal is to create a quiet beam, so we would like to consider the extent to which reducing shot noise will amplify energy noise. For example, FELs require energy spreads smaller than the Pierce parameter $\rho$, giving $\langle\Delta \eta\rangle \lesssim 10^{-3}$ for current x-ray FEL designs [22].

Quiet beams may be useful for controlling FEL start-up, which is driven by noise, $F(k)$, for SASE FELs, and from an external radiation field for seeded FELs. However, there is also a contribution to the FEL start-up from the energy noise [23], 


$$
F_{\eta}(k)=\frac{1}{N} \sum_{j, l} \frac{\hat{\eta}_{j} \hat{\eta}_{l}}{\rho^{2}} e^{i k\left[\hat{z}_{j}-\hat{z}_{l}\right]}
$$

We note that $F_{\eta}(k)$ scales as $\hat{\eta}^{2}$, which is always small. However, if $\hat{\eta}_{j}(z)$ is longitudinally periodic (as can be seen in Fig. 12 for $\left.k=k_{0}\right), F_{\eta}(k)$ will also scale as the number of particles $N$, which is generally very large. To claim a quiet start-up for an FEL, we must ensure that $F_{\eta}(k) \lesssim F(k)$.

[1] M. Borland, Y.C. Chae, P. Emma, J. W. Lewellen, V. Bharadwaj, W. M. Fawley, P. Krejcik, C. Limborg, S. V. Milton, H.-D. Nuhn, R. Soliday, and M. Woodley, Nucl. Instrum. Methods Phys. Res., Sect. A 483, 268 (2002).

[2] E. L. Saldin, E. A. Schneidmiller, and M. V. Yurkov, Nucl. Instrum. Methods Phys. Res., Sect. A 490, 1 (2002).

[3] S. Heifets, S. Krinsky, and G. Stupakov, Phys. Rev. ST Accel. Beams 5, 064401 (2002).

[4] Z. Huang and K.-J. Kim, Phys. Rev. ST Accel. Beams 5, 074401 (2002).

[5] R. Akre et al., Phys. Rev. ST Accel. Beams 11, 030703 (2008).

[6] D. Ratner, A. Chao, and Z. Huang, in Proceedings of FEL 2008, Gyeongju, Korea (Report No. SLAC-PUB-13392, 2008).

[7] A. Marinelli and J. B. Rosenzweig, Phys. Rev. ST Accel. Beams 13, 110703 (2010).

[8] E. L. Saldin, E. A. Schneidmiller, and M. Y. Yurkov, Opt. Commun. 202, 169 (2002).

[9] Z. Huang, in Proceedings of FEL 2006, Berlin, Germany (Report No. SLAC-PUB-12097, 2006).
[10] A. Gover and E. Dyunin, Phys. Rev. Lett. 102, 154801 (2009).

[11] A. Nause, E. Dyunin, and A. Gover, J. Appl. Phys. 107, 103101 (2010).

[12] V. Litvinenko, in Proceedings of the 31st International Free Electron Laser Conference (FEL 09), Liverpool, UK (STFC Daresbury Laboratory, Warrington, 2009).

[13] G. Stupakov, in Proceedings of FEL 2010, Malmo, Sweden (2010).

[14] K. Bane, in Workshop on the Physics and Applications of High Brightness Electron Beams, Erice, Italy (Report No. SLAC-PUB-11829, 2005).

[15] M. Venturini, Phys. Rev. ST Accel. Beams 11, 034401 (2008).

[16] M. Chodorow and C. Susskind, Fundamentals of Microwave Electronics (McGraw-Hill, New York, 1964).

[17] J. Rosenzweig, C. Pellegrini, L. Serafini, C. Ternieden, and G. Travish, Nucl. Instrum. Methods Phys. Res., Sect. A 393, 376 (1997).

[18] G. Stupakov and S. Krinsky, in Proceedings of the 20th Particle Accelerator Conference, Portland, OR, 2003 (IEEE, New York, 2003), pp. 3225.

[19] G. Geloni, E. Saldin, E. Schneidmiller, and M. Yurkov, Nucl. Instrum. Methods Phys. Res., Sect. A 583, 228 (2007).

[20] G. Geloni, E. Saldin, E. Schneidmiller, and M. Yurkov, DESY Report No. 05-032, 2005.

[21] J. B. Rosenzweig, M. P. Dunning, E. Hemsing, G. Marcus, P. Musumeci, and A. Marinelli, in Proceedings of FEL 2008, Gyeongju, Korea (2008).

[22] R. Bonifacio, C. Pellegrini, and L. M. Narducci, Opt. Commun. 50, 373 (1984).

[23] R. Bonifacio, F. Casagrande, and L. DeSalvo Souza, Phys. Rev. A 33, 2836 (1986). 